\title{
ABOUT THE NUMEROUS COST AND PROCESSING ADVANTAGES OF THE MICROCELLULAR FOAM INJECTION MOLDING PROCESS FOR THERMOPLASTICS MATERIALS IN THE AUTOMOBILE INDUSTRY
}

\author{
${ }^{1}$ A. Oprea-Kiss, ${ }^{2}$ I. Kiss \\ ${ }^{1}$ University Politehnica Timisoara, Faculty of Engineering Hunedoara, Romania, \\ ${ }^{2}$ University Politehnica Timisoara, Faculty of Engineering Hunedoara, Romania, \\ e-mail: imre.kiss@fih.upt.ro
}

\begin{abstract}
Today one of the goals of the automobile industry is to reduce weight. And physical foaming has already demonstrated its potential in this sector, improving the value and performance of applications under the bonnet: engine and gearbox cases, inlet air filters, cockpits, radiator baffles and so on. Around the world, the microcellular injection molding (MuCell) is used in thousands of applications in the automotive, packaging, technical molding, office machinery and electric and electronic component industries. The research opportunities purpose is to obtain even lighter pieces, with greater dimensional stability and with an excellent surface finish, in other words, perfect plastic parts. More component functionality with reduced weight, and cost control at the same time: MuCell is a process to physically foam thermoplastics, which combines technical and economic objectives. Besides weight reduction, it also provides improved dimensional stability of the moulded parts.
\end{abstract}

Keywords: microcellular injection molding (MuCell), supercritical fluid (SCF), thermoplastics, foaming, applications

\section{INTRODUCTION}

Microcellular injection molding (MuCell) is an innovative manufacturing technology in which supercritical (i.e., subject to extreme pressure) fluid (SCF) is dissolved into a polymer melt within a specially designed screw. [1, 3, 11-18] MuCell technology is a mold process technology, the patent rights to which are held by Trexel of the United States. In MuCell technology, supercritical gas is directly injected inside a screw cylinder and fused resin (liquefied gas) fills the inside of the mold. During injection, the SCF vaporizes and becomes gas bubbles (foam) within molded parts. Because the bubbles can in principle reach micron sizes, the process is known as microcellular foaming. MuCell offers many advantages over conventional injection technologies, such as less shrinkage, light weight, low materials cost, and fewer sink marks. In addition, MuCell-produced parts show less warping and closer tolerances, and in many cases also cool more quickly. [1, 3, 8-18] The microcellular injection molding (MuCell) is the most significant plastic processing innovation in the last 20 years. $[14,16]$

Compared to traditional injection molding processes, MuCell consists of the addition of precisely metered amounts of supercritical fluid (SCF) - typically nitrogen or carbon dioxide - into the polymer by means of injectors mounted on the plasticizing barrel. The specially designed mixing section of the screw finely disperses the SCF in a uniform manner in the melt. This solution has inferior viscosity to simple melt polymer, and therefore it flows more easily in the mold. When the fluid-polymer solution gets to the mold, where the pressure is lower than in the plasticizing barrel, the SCF begins to separate and expand, then micro cells begin to form. The expansion speed depends on the extent of the pressure drop: the higher it is, the greater the speed. The cell growth makes the mold cavity fill faster, because, as opposed to traditional molding, the SCF replaces the volume of material continually injected in the pack and hold phase to compensate for the shrinkage of the polymer in the cooling stage. [1,3,11-18]

In practice, the hold phase is no longer necessary as it is replaced by the inertial pressure of the SCF inside the piece, which pushes the polymer against the walls of the cavity filling it uniformly, even in the corners and the undercuts. This eliminates any great differences in pressure and temperature between the mold's injection point and the end point, generating a homogenous microcellular structure in the part, with less stress and strain and uniform shrink on every point of the surface and therefore greater compliances with tolerances. [9-18] 


\section{THE SUPERCRITICAL FLUIDS IN SOME THEORETICAL ASPECTS}

The supercritical fluids (SCF) are highly compressed gases at temperatures and pressures above the critical point. They combine properties of gases and liquids in a very intriguing manner. Due to the high compressibility of supercritical fluids, small changes in pressure can produce substantial changes in density which, in turn, affects diffusivity, viscosity and solvation properties of these fluids, thus dramatically influencing the kinetics and mechanisms of chemical reactions. [1-3]

A supercritical fluid is any substance at a temperature and pressure above its critical point, where distinct liquid and gas phases do not exist. It can effuse through solids like a gas, and dissolve materials like a liquid. In addition, close to the critical point, small changes in pressure or temperature result in large changes in density, allowing many properties of a supercritical fluid to be "fine-tuned". Many pressurized gases are actually supercritical fluids. $\mathrm{CO}_{2}$ and $\mathrm{N}_{2}$ are the most commonly used supercritical fluids. These are more often known as permanent gases. At room temperature, they are well above their critical temperature, and therefore behave as a gas, similar to $\mathrm{CO}_{2}$ at $400 \mathrm{~K}$ above. However, they cannot be liquefied by pressure unless cooled below their critical temperature.

Figure 1 show the pressure-temperature phase diagram, where the boiling separates the gas and liquid region and ends in the critical point, where the liquid and gas phases disappear to become a single supercritical phase. A small increase in pressure causes a large increase in the density of the supercritical phase. Many other physical properties also show large gradients with pressure near the critical point, e.g. viscosity, the relative permittivity and the solvent strength, which are all closely related to the density. At higher temperatures, the fluid starts to behave like a gas. Figure 2 show the carbon dioxide pressuretemperature phase diagram. At well below the critical temperature, e.g., $280 \mathrm{~K}$, as the pressure increases, the gas compresses and eventually (at just over 40 bar) condenses into a much denser liquid. The system consists of 2 phases in equilibrium, a dense liquid and a low density gas. [1-3, 7-10]

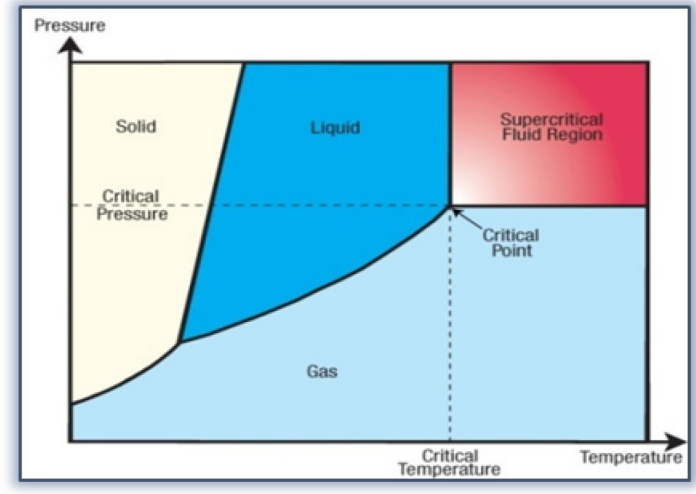

Figure 1. The supercritical fluid regions in the pressuretemperature phase diagram. [1-3, 11-18]

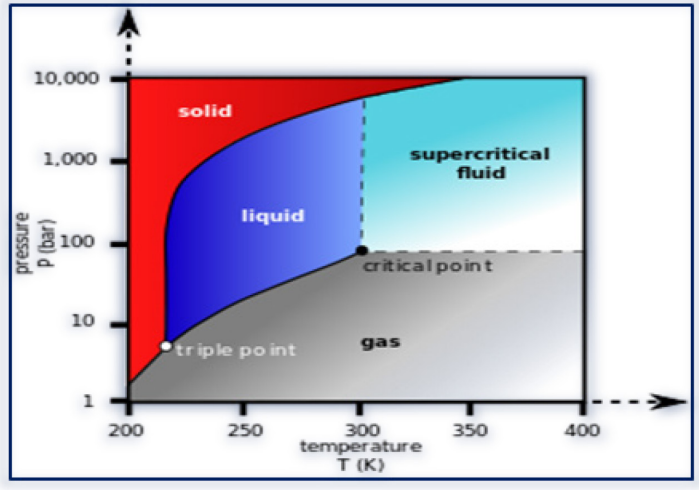

Figure 2. Carbon dioxide pressure-temperature phase diagram . [1-3, 11-18]

As the critical temperature is approached $(300 \mathrm{~K})$, the density of the gas at equilibrium becomes higher, and that of the liquid lower. At the critical point, $(\approx 300 \mathrm{~K}$ and $\approx 74$ bar $)$. There is no difference in density, and the two phases become one fluid phase. Thus, above the critical temperature a gas cannot be liquefied by pressure. At slightly above the critical temperature $(310 \mathrm{~K})$, in the vicinity of the critical pressure, the line is almost vertical. For carbon dioxide at $400 \mathrm{~K}$, the density increases almost linearly with pressure. Nitrogen has a critical point of $126 \mathrm{~K}$ and $\approx 34$ bar. Therefore, nitrogen (or compressed air) in a gas cylinder above this pressure is actually a supercritical fluid. [1, 11-18]

In addition, there is no surface tension in a supercritical fluid, as there is no liquid/gas phase boundary. By changing the pressure and temperature of the fluid, the properties can be "tuned" to be more liquid- or 
more gas-like. One of the most important properties is the solubility of material in the fluid. Solubility in a supercritical fluid tends to increase with density of the fluid (at constant temperature). Since density increases with pressure, solubility tends to increase with pressure. The relationship with temperature is a little more complicated. At constant density, solubility will increase with temperature. However, close to the critical point, the density can drop sharply with a slight increase in temperature. Therefore, close to the critical temperature, solubility often drops with increasing temperature, then rises again. [1, 7, 11-18] All supercritical fluids are completely miscible with each other so for a mixture a single phase can be guaranteed if the critical point of the mixture is exceeded. [1-3, 11-18]

\section{OVERVIEW OF MICROCELLULAR FOAMING TECHNOLOGY PROCESS}

In the Microcellular Foaming Technology (MuCell) process, $\mathrm{CO}_{2}$ or nitrogen is injected as supercritical fluid in the last part of the extruder. The gas molecules are then immediately dissolved in the melted polymer and stay in the melt until there is a pressure drop in the extrusion head. $[1-3,11-18]$

The MuCell consists of the addition of supercritical fluid (SCF) into the polymer by means of injectors mounted on the plasticizing barrel. The specially designed mixing section of the screw finely disperses the SCF in a uniform manner in the melt. Supercritical fluids (SCF) are fluids above a critical point characterized by their critical temperature and pressure values. A supercritical fluid is substantially a dense gas, with intermediate properties between a gas and a liquid which depend significantly on its density. The special properties of a SCF are determined by the marked dependence of density on the temperature and pressure conditions close to the critical point. The importance of SCF lies in the fact that it is possible to fine-tune its properties within a wide range though small changes in temperature and pressure values. It should be noted that any gas above its critical temperature is in an incoercible gas phase, thereby it cannot be liquefied simply by pressure. [1-3, 11-18]

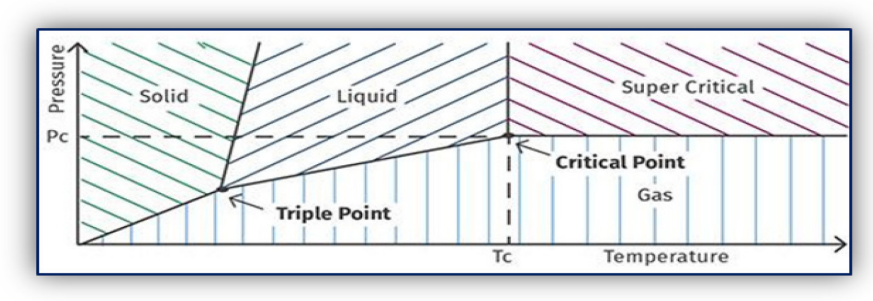

Figure 3. How the MuCell process works. [1-3, 11-18]

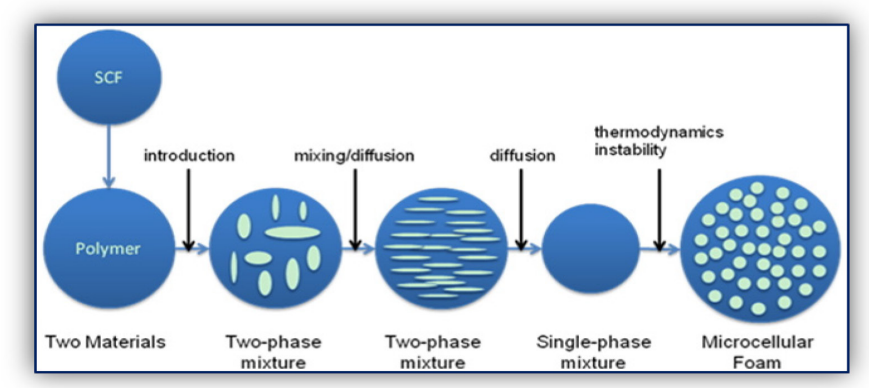

Figure 4. The Microcellular Foaming Technology process (the addition of supercritical fluid). [1-3, 11-18]

The microcellular technological process consists of four main, sequential steps, which end with solidification. [1-3, 6-7, 11-18] 
The four main steps are:

$\equiv$ Gas dissolution - In the plastication period of the microcellular injection molding process, a supercritical fluid (SCF) or blowing agent, nitrogen $\left(\mathrm{N}_{2}\right)$ or carbon dioxide $\left(\mathrm{CO}_{2}\right)$, is injected in to the polymer to form a single-phase solution. The gas is dissolved in the polymer melt due to applied high pressure.

$\equiv$ Nucleation - this is the "seeding" of bubbles and occurs as a result of a pressure drop. There are two types of processes, either short shot or full shot:

$\equiv$ Short-shot process - The mixture is injected into the mold cavity as a short shot to fill only part of the cavity. Due to the substantial and rapid pressure drop, the solution of the gas in the melt becomes supersaturated and a large number of bubbles nucleate.

$\equiv$ Full-short process - The mixture is injected to fill the mold cavity completely. After the cavity is volumetrically filled, it is pressurized and the nozzle is shut off. As the material within the cavity cools and then shrinks, bubbles nucleate due to the pressure falls.

$\equiv$ Bubble growth - this is the increase in size of the bubble seeds as the gas diffuses out of the melt and into the bubbles.

$\equiv$ Solidification - During the foaming process the mold is continuously cooled down, creating the internal cellular structure. The result is a foamed material with cell size of around 5-100 microns, the actual size depending on processing conditions. The lack of a formal packing phase reduces the residual stress in the material and results in extremely low warpage.

$\equiv$

a)
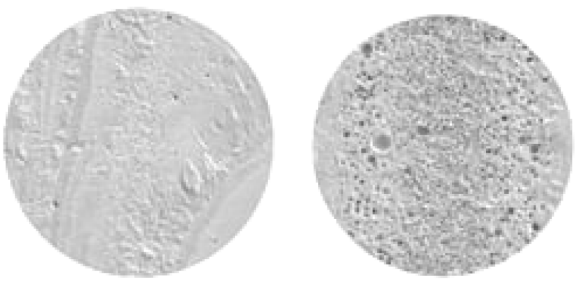

b)

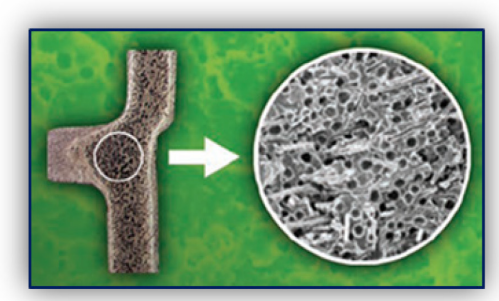

Figure 6. The microcellular foaming [11-18]

Figure 5. The microcellular foaming structure. [11-18] a) before; b) after

The physical foaming of polymers promises a number of advantages in injection molding processes. The structural foam molding technology taking advantage of the physical foaming of polymers in the mold, better known as the MuCell process, is far from new. After almost a decade since the first industrial applications were launched on the market, this technology seems to have found new vitality in the automotive, technical and packaging component markets, to which it delivers light weight without compromising the stability of the manufactured item.

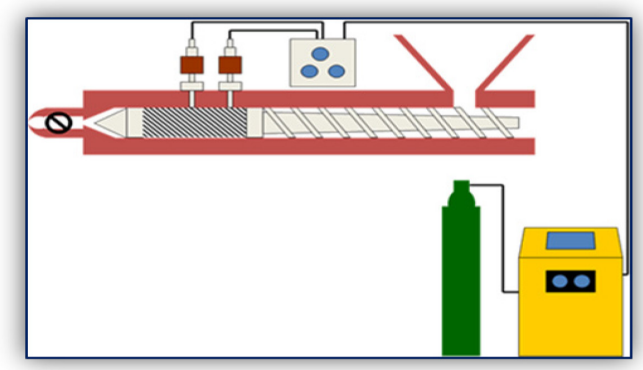



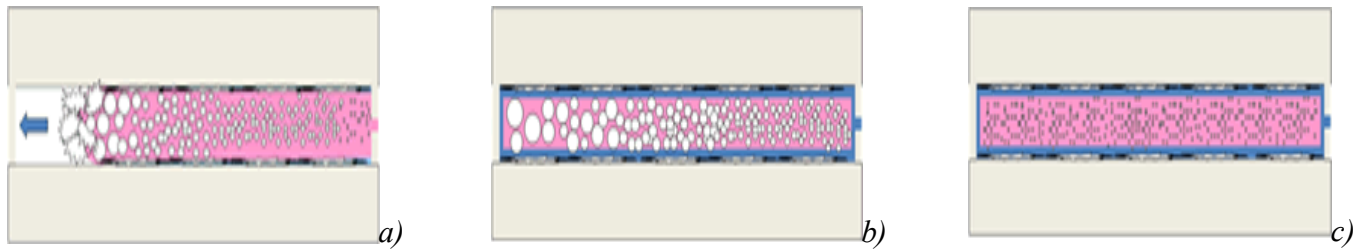

Figure 8. Model of behavior of cells in mold in MuCell: a) Condition of cells in the middle of filling; b) Condition of cells after reaching the end; c) Condition of cells when the internal pressure rises [1-3, 11-18]

An overview of MuCell is as shown above. [1-3, 11-18] Supercritical fluids $\left(\mathrm{CO}_{2}\right.$ and $\left.\mathrm{N}_{2}\right)$ are injected into a cylinder to make a single-phase solution, and the single-phase polymer solution is injected into a mold. In the mold, the pressure drops rapidly to generate huge numbers of cell nucleation. In the forming material containing supercritical fluids, cells expand, and the volume of the material increases. The mold cavity is filled with the material, which flows in it. The diameters of the cells increase as the distance away from the gate. At the flow end, cells break violently and release gas. When the filling quantity increases and the internal pressure rises, the cells are compressed, and the cell sizes become smaller. [11-18]

Solved physical foaming agent $\left(\mathrm{CO}_{2}\right.$ or $\left.\mathrm{N}_{2}\right)$ becomes cells. When cells break, traces of broken gas bubbles contact the inner surface of the mold cavity. If the mold temperature is low at this time, the traces of broken gas bubbles cool and remain on the article surface. In processing with much foam gas like MuCell, cells that will break are produced in large quantity and make patterns along the direction of flow of the foaming material. [1-3, 11-18] These patterns may look like eddies and are called swirl marks.

\section{THE ADVANTAGES OF THE MuCell TECHNOLOGY}

The MuCell microcellular foam injection molding process for thermoplastics materials provides unique design flexibility and cost savings opportunities not found in conventional injection molding. The MuCell process allows for plastic part design with material wall thickness optimized for functionality and not for the injection molding process. The combination of density reduction and design for functionality often results in material and weight savings of more than $20 \%$.

The major advantage of MuCell is that it facilitates the injection molding operations compared to the traditional process, which poses the challenge of making the polymer flow from the injection point until the end of the mold cavity without cooling it, and to compact it in the most uniform way possible to obtain a uniform shrink, thus boosting dimensional stability and avoiding problems such as sink marks and warpage.
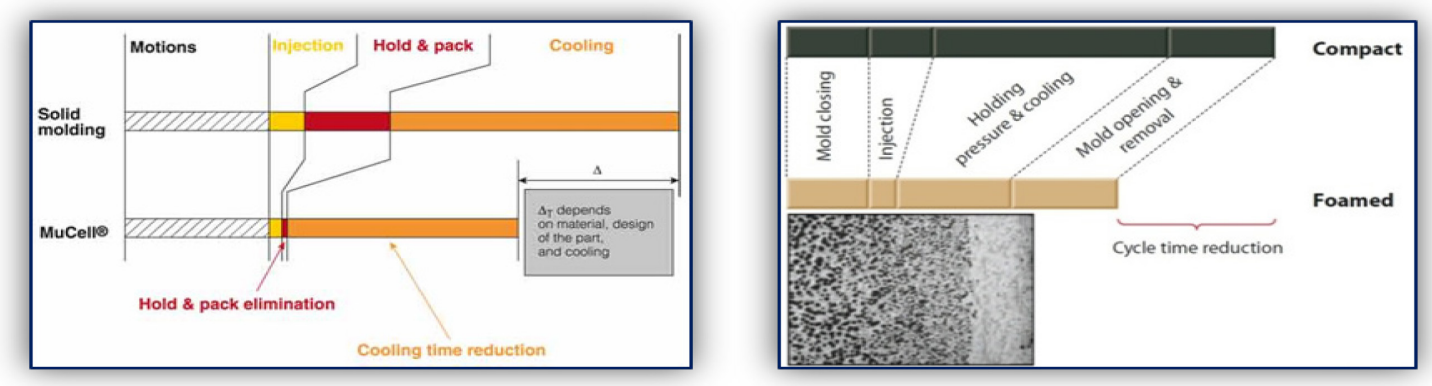

Figure 9. Advantages of the MuCell (graphically representation) [1-3, 11-18]

The reduction in viscosity, which can be as high as 30\% (10-15\% in glass fiber filled melts), improves the melt flow index (MFI) of the material, which flows faster thereby reducing the injection time. Along with 
the fact that the mold can be kept at a lower temperature and that the hold phase is eliminated, all this contributes greatly also to reducing the cycle time by as much as $50 \%$. In addition, the improved MFI of the melt means operating at a lower pressure, and therefore with a significantly lower clamping force (up to $50 \%$ less). Smaller machines are more affordable, take up less space and consume less energy, three determining factors for efficiency in the production process.

The MuCell process, have these general observed advantages: [1-3, 11-18]

$\equiv$ getting rid of material waste;

$\equiv$ lowering cycle times;

$\equiv$ lightening part weights;

$\equiv$ higher dimensional precision.

The cell expansion inside the mold also has many advantages. The weight of the piece is reduced and fewer polymers is needed, as it is replaced by the micro-cells that form a foam structure and show a mechanical strength very similar to human bones.

To enhance innovative output, conventionally manufactured plastic components have to compete again and again. The customer's demands are clearly defined: more functionality with reduced weight, and cost control at the same time. The only way to correctly interpret these market demands and offer solutions that are economical in the long run is to embrace new technologies in a responsible and efficient manner.

By replacing the tradition pack \& hold phase of the solid molding process with cell growth, the MuCell process allows for uniform and locally applied pack pressure through cell growth. [1-3, 11-18] Gate locations are then placed in the thin cross sections for optimized filling patterns while allowing the cell growth to provide packing in the thicker cross sections at the end of fill. By replacing the pack \& hold phase, lower stress parts are produced which have enhanced dimensional stability and substantially reduce warpage. This allows part design to be optimized with material thickness in those areas that require strength and reduced wall thickness in areas that are not structural. Cell growth also results in the elimination of sink marks. Unlike chemical foaming agents, the physical MuCell process has no temperature limitation and does not leave any chemical residue in the polymer, making consumer products perfectly suitable for recycling and allowing re-grind material to reenter the process flow.

The microcellular injection molding, commercially known as the MuCell process, is capable of massproduction with complex geometry and excellent dimensional stability. In this process, Supercritical Fluid (SCF), usually as nitrogen $\left(\mathrm{N}_{2}\right)$ or carbon dioxide $\left(\mathrm{CO}_{2}\right)$, is mixed with polymer melt to create a uniform single-phase solution, then being injected into the mold cavity. [1-3, 11-18] The melt flow with bubbles will foam and form a part.

MuCell technology is a process consisting in the injection of a gas into the fused polymer, which in its turn expands itself providing the hereunder advantages: [1-3, 11-18]

$\equiv$ significant weight savings ( $8-15 \%$ density reduction and weight saving through new design options);

$\equiv$ reduction of inner cavity pressure and clamp tonnage (30-50\%);

$\equiv$ reduction of material viscosity;

$\equiv$ reduction or elimination of hold time;

$\equiv$ significant reduction in process temperature;

$\equiv$ reduction of overall cycle time;

$\equiv$ eliminates sink marks in the end product.

The advantages of the MuCell process at a glance:

$\equiv$ economical: Production on machines with lower locking force;

$\equiv$ reliable: Reproducible machine parameters;

$\equiv$ light: Component density reduction;

$\equiv$ dimensional stability: Injected gas ensures reduced distortion;

$\equiv$ quick dimensional accuracy: Thanks to additional pressure in the tool.

Therefore, in summary, the advantages of the Microcellular Injection Molding Process are:

$\equiv$ highly enhance the dimensional accuracy on product; 
$\equiv$ reduce product weight without reducing the structural strength;

$\equiv$ reduce cycle time;

$\equiv$ minimize non-uniform shrinkage and warpage;

$\equiv$ achieve part lightweight and environmental friendliness,

$\equiv$ extend product life cycle.

The numerous cost and processing advantages have led to rapid global deployment of the MuCell process primarily in automotive, consumer electronics, medical device, packaging and consumer goods applications. The MuCell application main advantage is the freedom to design for functionality and not for plastic process limitations.

\section{THE DISADVANTAGES OF THE MuCell TECHNOLOGY}

Despite these benefits, widespread application of MuCell is hampered by inadequate control over the microcellular foaming process. When foaming occurs along the melt front, advancement frequently introduces streaklike flow marks on the molded surface. [2, 11-18] These imperfections can be remedied using co-injection or in-mold decoration technology, but at a high cost. The SCF foaming also causes changes in melt viscosity and other physical properties. This leads to no uniform bubble sizes, which limits the use of MuCell for optical parts such as light guide plates that rely on diffusion.

Here, a gas-counter pressure (GCP) method is described that controls foaming by applying different gas pressures at the melt-injection stage (Figure 9). [1-3, 11-18] The injection speed is determined by the difference between the screw pressure $\left(\mathrm{P}_{\text {screw }}\right)$ and that of the gas $\left(\mathrm{P}_{\text {gas }}\right)$. When $\mathrm{P}_{\text {screw }}$ is slightly higher than $\mathrm{P}_{\text {gas }}$, and both parameters are high enough, the SCF-dissolved melt flows into the mold cavity without foaming. Setting $\mathrm{P}_{\text {screw }}$ higher than $\mathrm{P}_{\text {gas }}$, and $\mathrm{P}_{\text {gas }}$ lower than the critical pressure, results in partial foaming. Finally, the appropriate choice of $\mathrm{P}_{\text {screw }}, \mathrm{P}_{\text {gas }}$, and pressure difference combined with dynamic mold temperature enables more precise control of bubble size. A preliminary test shows that it is quite possible to eliminate flow induced streaks using the GCP technique. How foaming affects the viscosity of the melt with dissolved SCF, is also measured. $[4,5,9,10]$
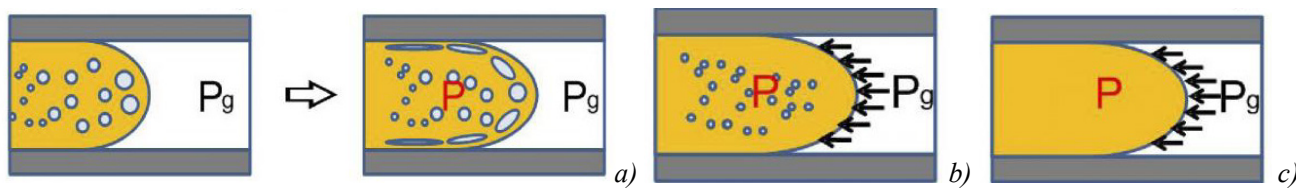

Figure 10. Schematic drawing of the microcellular injection molding (MuCell) process using gas counter pressure (GCP);

P: Pressure; Pg: Gas pressure; a) microcellular injection molding (MuCell) process, without GCP technique, Pg=0; b) microcellular injection molding (MuCell) process, with GCP technique (partial foaming), $P>P g, P g<P c r i t i c a l ; c)$ microcellular injection molding (MuCell) process, with GCP technique (no foaming), P $>$ Pg, Pg $>$ Pcritical

The main industrial challenges are: [1-3, 8, 11-18]

$\equiv$ different designs of part geometry, part wall, and rib thickness from the traditional foaming process;

$\equiv$ optimal combination mix of polymer and SCF;

$\equiv$ controls of process condition variations, such as temperature or pressure;

$\equiv$ effects of gate number and location on welding lines and air traps;

$\equiv$ potential surface quality problems.

Finally, what are the benefits of the microcellular injection molding? They are:

$\equiv$ most engineering and filled polymers are able to be processed without significant compromise to performance or durability; 
$\equiv$ the added benefits achieved are greater stability, less built-in stress, and a resultant reduction in warp and distortion. With the vertical MuCell molding capabilities, complex insert molded parts using delicate traces and difficult fill patterns can typically be achieved with less scrap and better control;

$\equiv$ due to the lower pressures and softer fills, delicate items can be overmolded without much of the traditional displacement and resultant need for excessive control features. This simplifies molds and makes the process and product more robust;

$\equiv$ in straight molding applications, reduced weight, faster cycles and less distortion result in better product quality and cost. All this is achieved without the need for expensive custom plastic materials or additives and without the uncertainty of large voids or other internal defects.

\section{TECHNOLOGIAL REMARKS / CONCLUSIONS}

The MuCell process for producing microcellular injection molded parts is used and accepted globally as a technology option for providing a more dimensionally stable part through a reduction in residual stress, along with increased productivity versus solid injection molded parts.

The trend in structural-foam molding is toward larger, more productive presses that produce multiple parts per cycle. Machines equipped with multiple extruders, not only mold two different colored parts on the same press, but also run two different materials or structural foam and structural web gas-assist parts simultaneously. The process works with most thermoplastics and low cavity pressure lets designers specify lower-cost aluminum molds. This is a benefit for small production runs that may otherwise be too costly to produce via conventional injection molding with expensive tool steel molds. Therefore, the structural-foam molding can produce hollow, multilayered parts that are often bigger, stronger, lighter, and cost less than injection-molded equivalents.

Designing for function means putting material where it needs to be and not simply to assist in filling and packing out the part. In microcellular foaming, weight reduction is achieved as much or more through changes to the nominal wall thickness as it is from density reduction. In most cases, a part designed for function ends up with less density reduction than the same part design for solid molding and converted to foam, but with a much greater overall weight reduction.

Therefore, this technology is used for the production of high performance existing and new products with unique characteristics in regard of composition (purity), size (micro or nanoscale) and architecture (fibers, foams). The MuCell process for producing microcellular injection molded parts is used and accepted globally as a technology option for providing a more dimensionally stable part through a reduction in residual stress, along with increased productivity versus solid injection molded parts.

\section{REFERENCES}

[1] D. F. Baldwin, D. E. Tate, C. B. Park, S. W. Cha, N. P. Suh, Microcellular plastics processing technology, J. Japan Soc. Polym. Process, 6, (1994), pp. 187-256.

[2] L. S. Turing, H. Kharbas, A novel microcellular co-injection molding process, Proc. ANTEC, (2004), pp. 535-539

[3] S. C. Chen, R. P. Yang, Y. W. Lin, P. S. Hsu, S. S. Hwang: Study on pressure control device to improve foaming uniformity for the injection molding microcellular foaming process, Proc. ANTEC, pp. 699-703, 2007.

[4] J. R. Royer, J. M. Desimone, S. A. Khan, High pressure rheology and viscoelastic scaling predictions of polymer melts containing liquid and supercritical carbon dioxide, J. Polym. Sci. B Polym. Phys. 39, pp. 3055-3066, 2001

[5] C. Kwag, C. W. Manke, E. Gulari, Rheology of molten polystyrene with dissolved supercritical and near-critical gases, J. Polym. Sci. B Polym. Phys., 37 (19), (1999), pp. 2771-2781

[6] C. B. Park, D. F. Baldwin, N. P. Suh, Effect of the pressure drop rate on cell nucleation in continuous processing of microcellular polymers, Polymer Engineering \& Science, 35 (5), (1995), pp. 432-440 
[7] K. A. Arora, A. J. Lesser, T. J. McCarthy, Preparation and characterization of microcellular polystyrene foams processed in supercritical carbon dioxide, Macromolecules, 31 (14), (1998), pp. 4614-4620

[8] A. N. Spoerrer, D. S. Bangarusampath, V. Altstaedt, The challenge of foam injection-moulding: possibilities to improve surface appearance, foam morphology and mechanical properties. Cellular polymers, 27(2), (2008), pp. 101-121

[9] C. A. Villamizar, C. D. Han, Studies on structural foam processing II. Bubble dynamics in foam injection molding, Polymer Engineering \& Science, 18 (9), (1978), pp. 699-710

[10]K. A. Arora, A. J. Lesser, T. J. McCarthy, Preparation and Characterization of Microcellular Polystyrene Foams Processed in Supercritical Carbon Dioxide, Macromolecules, 1998, 31 (14), pp. 4614-4620

[11] http://www.plastix-world.com/the-power-of-bubbles/

[12] http://greenmolding.org/english/solutio

[13] http://www.moldex3d.com/en/products/solution-addons/mucell

[14] http://www.trexel.com/en/mucell-injection-molding (http://www.trexel.com/en/)

[15] http://www.alplastics.net/ALPlastics_MuCell.pdf

[16] http://www.mucell.com/

[17] http://www.engelglobal.com/en/ro/solutions/technologies/foammelt.htm

[18] http://www.negribossi.com/technologies/mucell-microcellular-foam/ 